\title{
Assimilation of Satellite Radiances for Numerical Weather Prediction
}

\author{
Michael D. Tsyrulnikov ${ }^{1}$, Dmitry R. Gayfulin ${ }^{1}$, Alexander B. Uspensky ${ }^{2}$ \\ ${ }^{1}$ Hydrometcenter of Russia, Moscow, Russia \\ tsyrulnikov@mecom.ru \\ ${ }^{2}$ SRC "Planeta", Moscow, Russia \\ uspensky@planet.iitp.ru
}

\begin{abstract}
General data assimilation methodology is outlined. Variational, ensemble based, and hybrid ensemble-variational approaches currently used in operational weather forecasting are briefly reviewed. Their advantages and disadvantages are listed. Specificity of satellite radiance data assimilation is discussed, including roles of observation-error covariance matrix, observation operator, and bias correction, and impact of clouds and precipitation. Estimates of the accuracy of microwave radiances from the MTVZA-GY radiometer and their impact on the data assimilation system of the Hydrometcentre of Russia are given. A significant positive impact on three-day weather forecasts in the southern hemisphere in the absence of AMSU-A data is found. Observations produced by the infrared Fourier spectrometer IKFS-2 onboard Meteor-M N2 are also examined. Preliminary results on their accuracy and on their impact on assimilation are presented.
\end{abstract}

Keywords: data assimilation, satellite observations, microwave radiances, infrared radiances, bias correction, MTVZA-GY, IKFS-2. 


\title{
УСВОЕНИЕ СПУТНИКОВЫХ РАДИАЦИОННЫХ НАБЛЮДЕНИЙ В ЗАДАЧЕ ЧИСЛЕННОГО ПРОГНОЗА ПОГОДЫ
}

\author{
М.Д. Цырульников ${ }^{1}$, Д.Р. Гайфулин ${ }^{1}$, А.Б. Успенский \\ ${ }^{1}$ Гидрометцентр России, Москва, Россия \\ tsyrulnikov@mecom.ru
}

${ }^{2}$ Научно-исследовательский центр космической гидрометеорологии «Планета», Москва, Россия uspensky@planet.iitp.ru

Даётся краткое введение в общую методологию усвоения данных. Кратко описываются вариационные, ансамблевые и гибридные вариационно-ансамблевые методы усвоения данных. Перечислены их достоинства и недостатки. Обсуждаются особенности усвоения данных радиационных спутниковых наблюдений, включая роль матрицы ковариаций ошибок наблюдений, оператора наблюдений и коррекции смещений, а также влияния облаков и осадков. Приводятся результаты анализа точности измерений микроволнового радиометра МТВЗА-ГЯ и их вклада в систему усвоения данных Гидрометцентра России. Выявлен существенный положительный эффект от усвоения данных МТВЗА-ГЯ на трёхсуточный прогноз погоды в южном полушарии в отсутствие данных AMSU-A. Также проанализированы данные измерений инфракрасного фурье-спектрометра ИКФС-2 на борту спутника Метеор-М №2. Даются предварительные результаты анализа их точности и усвоения данных.

Ключевые слова: усвоение данных, спутниковые наблюдения, микроволновые радиации, инфракрасные радиации, коррекция смещений, МТВЗА-ГЯ, ИКФС-2

\section{Введение}

Спутниковые наблюдения состояния земной атмосферы и подстилающей поверхности являются в настоящее время ключевым компонентом глобальной метеорологической наблюдательной системы. По вкладу в качество численного прогноза погоды спутниковые наблюдения доминируют в южном полушарии и соизмеримы с традиционными контактными наблюдениями (наземные станции, корабли и буи, радиозонды, самолётные наблюдения) в северном полушарии.

Важнейшим видом спутниковых наблюдений являются измерения уходящего излучения системы «Земля-атмосфера» в микроволновом и инфракрасном диапазонах спектра электромагнитных волн. Эти наблюдения чувствительны к температуре и влажности в атмосфере. Достоинством микроволновых наблюдений является их всепогодность: они не подвержены влиянию облаков, за исключением наиболее мощных. Достоинство инфракрасных наблюдений - их более высокое (по сравнению с микроволновыми наблюдениями) горизонтальное и вертикальное разрешение.

Спутниковые и другие наблюдения используются при численном прогнозе погоды с помощью технологии усвоения данных. В данной статье мы кратко охарактеризуем методы и подходы усвоения данных, дадим представление о специфике усвоения спутниковых радиационных наблюдений, а также представим результаты анализа точности и результаты усвоения данных микроволнового радиометра МТВЗА-ГЯ и инфракрасного фурье-спектрометра ИКФС-2 в системе глобального усвоения данных Гидрометцентра России. 


\section{Методика усвоения данных наблюдений}

Задачей усвоения данных является формирование возможно более точной оценки текущего состояния «системы» (в нашем случае атмосферы) по данным наблюдений. Эта оценка служит начальными данными для старта прогностической модели. Формирование оптимальной оценки состояния атмосферы (называемой в метеорологии и океанологии анализом) сопряжено со следующими трудностями.

Во-первых, наблюдения, как правило, имеют недостаточную густоту в пространстве для восстановления состояния атмосферы с требуемым разрешением по горизонтали и вертикали. Наблюдения также расположены зачастую весьма нерегулярно. В результате необходима интерполяция или экстраполяция в области атмосферы не покрытые наблюдениями. Во-вторых, наблюдения содержат ошибки. Ошибки бывают систематические и случайные. Обусловлены они неточностью измерительных приборов, ошибками при кодировке измерений, ошибками т.н. оператора наблюдений (см. ниже), а также возможной нерепрезентативностью наблюдений (когда наблюдаются компоненты атмосферных полей не разрешаемые на сетке анализа). Втретьих, наблюдаться могут не все интересующие нас метеоэлементы или не все в равной мере. В-четвертых, наблюдаться могут совсем не те характеристики атмосферы, которые нас интересуют. В-пятых, при анализе состояния атмосферы в данный момент времени было бы разумно использовать не только текущие, но и прошлые наблюдения - имея в виду инерционность атмосферных процессов. Задача привлечения информации, содержащейся в прошлых наблюдениях, решается с использованием прогностической модели атмосферы.

Таким образом, усвоение данных наблюдений - это совокупность научных и технологических методов и подходов, направленных на эффективное извлечение информации из разнообразных (прямых и косвенных, контактных и дистанционных) наблюдений параметров состояния той или иной среды (в данном случае - атмосферы) с использованием её математической модели.

Оптимизация процесса усвоения данных осуществляется путём введения вероятностных моделей всех источников неопределённости, включая ошибки наблюдений и пространственно-временные поля ошибок прогностической модели, с использованием методов оптимальной фильтрации. Технологически усвоение данных производится с помощью т.н. последовательного (рекуррентного, циклического) подхода шагами по времени (в атмосфере - это обычно от 1 до 6 часов). При этом на каждом шаге оценка состояния атмосферы («анализ») формируется путем оптимального сочетания текущих наблюдений и численного прогноза, стартовавшего с анализа на предыдущем шаге усвоения (т.н. первого приближения).

\section{Вариационные методы усвоения данных}

Вариационные методы основаны на минимизации функционала качества, который представляет собой квадратичную норму отклонения искомого поля анализа $X^{a}$ от вектора первого приближения $X^{f}$ и вектора наблюдений $X^{o}$ :

$J\left(X^{a}\right)=\left(X^{a}-X^{f}\right)^{T} B^{-1}\left(X^{a}-X^{f}\right)+\left(H\left(X^{a}\right)-X^{0}\right)^{T} R^{-1}\left(H\left(X^{a}\right)-X^{o}\right) \rightarrow \min$

где $H$ - т.н. оператор наблюдения, а весовые матрицы двух квадратичных форм оптимизируются путём их задания как обратных ковариационных матриц ошибок первого приближения (В) и ошибок наблюдений $(R)$.

Различают 2 основных подхода: трёхмерное и четырёхмерное вариационное усвоение (3D-Var и 4D-Var). При 3D-Var оператор наблюдения 4D-Var связывает усваиваемые наблюдения непосредственно с анализируемыми полями:

$$
X^{o}=H(X)+\eta
$$

где X - искомое истинное состояние атмосферы на сетке анализа, а $\eta$ - вектор ошибок наблюдений (включающий ошибки собственно измерения и ошибки оператора $H$ ). 
При 4D-Var оператор $H$ включает в себя не только связь наблюдений с анализируемыми полями в данный момент времени, но и прогностическую модель $M$, связывающую поля в моменты времени наблюдений с полями в начальный момент времени т.н. окна усвоения, где и ищутся поля анализа: $H=H^{3 D V a r} \cdot M$.

Достоинством вариационных методов усвоения данных является их способность эффективно усваивать косвенные и нелокальные наблюдения, в первую очередь спутниковые. Оперативная система усвоения данных Гидрометцентра России основана на методе 3D-Var [1].

\section{Ансамблевые и гибридные методы усвоения данных}

Вариационные подходы традиционно предполагают, что матрица ковариаций ошибок первого приближения В не зависит от времени, что является не вполне адекватной моделью реальности, где матрица В оказывается зависящей как от изменчивой структуры атмосферного потока, так и от вариаций в составе вектора наблюдений. Этот недостаток преодолевается в схемах ансамблевого фильтра Калмана, в которых матрица В не задаётся априори (как в вариационных схемах) и не вычисляется явно (как в классическом фильтре Калмана), а оценивается по выборке Монте-Карло. Такой подход оказался весьма продуктивным (и существенно более простым, чем вариационный). Однако объём выборки Монте-Карло (т.н. ансамбля) не может быть в реальных задачах достаточно большим для получения надёжных статистических оценок матрицы ковариаций. Её оценки неизбежно оказываются зашумленными и имеющими низкий ранг. Первое снижает точность анализа, а второе позволяет эффективно усваивать только относительно небольшие объёмы наблюдений.

Для того чтобы статистическая оценка матрицы ковариаций стала применимой для усвоения данных наблюдений, применяются многочисленные методы её регуляризации. Наиболее популярным методом является т.к. локализация, когда или обнуляются достаточно далёкие ансамблевые ковариации, или когда анализ делается локально с ограниченным набором «влияющих наблюдений» (как в методе оптимальной интерполяции). Другой метод регуляризации - смешивание ансамблевых и статических ковариаций - ведёт к гибридным вариационно-ансамблевым методам, считающимся в настоящее время наиболее перспективными. В [2] был предложен метод гибридизации с использованием скользящего сглаживания ковариаций во времени. Используются и другие, в основном эмпирические, подходы - инфляция ковариаций, пространственное осреднение ковариаций и др.

\section{Усвоение спутниковых радиационных наблюдений}

Наибольшие успехи в усвоении радиационных наблюдений были достигнуты с помощью вариационных и вариационно-ансамблевых подходов. Для эффективного усвоения таких наблюдений необходимы, как это следует из уравнения (1), не только матрица $B$ (задание которой обсуждалось выше), но матрица ковариаций ошибок наблюдений $R$, а также оператор наблюдений $H$.

Касаемо матрицы $R$, в случае радиационных наблюдений обычно используемое упрощающее предположение о её диагональности часто оказывается невыполненным. Так в [3] обнаружены значительные пространственные, меж-канальные и меж-спутниковые корреляции ошибок наблюдений радиометра AMSU-A. B [4] показано, что введение ненулевых меж-канальных корреляций для наблюдений интерферометра IASI дало положительный эффект в усвоении этих данных.

Оператором радиационных наблюдений служит модель переноса излучения в атмосфере. Эта модель должна быть достаточно быстрой (учитывая огромные объёмы спутниковых данных), а также достаточно точной (её ошибки приплюсовываются к ошибкам измерения). В $[1,3,5]$ мы использовали модель RTTOV [6]. 
Важнейшую роль при усвоении спутниковых радиационных наблюдений играет учёт влияния облаков и осадков. В настоящее время в ведущих прогностических центрах мира происходит постепенный переход от традиционного подхода с отбрасыванием облачных сцен (clear-sky) к подходу с учётом влияния полей гидрометеоров (в облаках и осадках) - в первую очередь, для микроволновых, а затем и для инфракрасных наблюдений (all-sky). Подход all-sky позволяет существенно повысить информативность радиационных наблюдений чувствительных к гидрометеорам [7].

Неотъемлемым компонентом системы усвоения радиационных спутниковых наблюдений является т.н. коррекция смещений (систематических ошибок). Необходимость такой коррекции обусловлена тем, что методы оптимального усвоения (см. выше) предполагают несмещённость ошибок наблюдений. Наиболее простым подходом является сравнение данных наблюдений с полями первого приближения, к которым применён оператор наблюдений $H$. При этом оцениваются и затем применяются параметры достаточно простой модели смещений [8]. Мы следовали этому подходу в [1,3,5]. Более совершенным является т.н. вариационная коррекция смещений [9], при которой параметры (линейной) модели коррекции включаются в вектор анализа $X^{a}$ и оцениваются аналогично вектору состояния атмосферы в циклической схеме усвоения данных.

\section{Анализ точности данных измерений МТВЗА-ГЯ}

Микроволновый радиометр МТВЗА-ГЯ установлен на борту спутника Метеор-М №2 [10]. Его радиометрические каналы имеют рабочие частоты в окнах прозрачности атмосферы 10,6, 18,7, 23,8, 31,5, 36,7, 42, 48 и 91 ГГц (сканер), а также в линиях поглощения кислорода 52-57 ГГц (температурное зондирование) и водяного пара 183 ГГц (влажностное зондирование). Полоса обзора прибора составляет 1500 км, пространственное разрешение - 16-198 км (в зависимости от канала).

В [5] мы оценили точность измерений радиационной температуры радиометра МТВЗА-ГЯ путём их сравнения с яркостными температурами, вычисленными с помощью модели RTTOV по данным 6-часового прогноза NCEP. Результаты (среднеквадратичные отклонения, К) представлены в следующей таблице.

\begin{tabular}{|l|c|c|c|c|c|c|c|c|c|}
\hline & \multicolumn{5}{|c|}{ Температурные каналы } & \multicolumn{3}{|c|}{ Влажностные каналы } \\
\hline Данные\каналы & Ch15 & Ch16 & Ch17 & Ch18 & Ch19 & Ch20 & Ch27 & Ch28 & Ch29 \\
\hline Без коррекции & 7.53 & 13.3 & 15.4 & 13.5 & 6.65 & 10.5 & 18.4 & 18.5 & 13.8 \\
\hline Коррекция [8] & 0.67 & 0.83 & 1.70 & 0.62 & 0.71 & 0.79 & 2.00 & 2.15 & 2.65 \\
\hline $\begin{array}{l}\text { Коррекция по } \\
\text { солн. углам [5] }\end{array}$ & 0.56 & 0.63 & 1.04 & 0.50 & 0.56 & 0.62 & 1.91 & 2.01 & 2.38 \\
\hline
\end{tabular}

Здесь столбцы соответствуют каналам MTB3А-ГЯ (в нумерации RTTOV). 1-я строка показывает результаты без коррекции смещений (видны чрезвычайно большие ошибки - в основном за счёт глобальных смещений). Во 2-й строке представлены результаты после схемы коррекции [8] (ошибки стали радикально меньше), а в 3-ей строке - после применения разработанной нами схемы адаптивной коррекции с учётом солнечных углов [5] (ошибки стали ещё меньше). По сравнению с данными AMSU-A и MHS, проанализированными за тот же период времени, ошибки в температурных и влажностных каналах МТВЗ-ГЯ оказались в среднем примерно в 1.52 раза больше, чем в аналогичных каналах AMSU-A и MHS. 


\section{Результаты усвоения данных измерений МТВЗА-ГЯ}

Численные эксперименты по усвоению данных наблюдений МТВЗА-ГЯ в системе глобального усвоения данных Гидрометцентра России показали результаты, представленные на рис.1 (подробнее - см. [1]). В северном полушарии эффект от внедрения наблюдений МТВЗА-ГЯ оказался нейтральным. В южном полушарии эффект (в отсутствии наблюдений AMSU-A) оказался ощутимо положительным и составил примерно половину эффекта от усвоения данных AMSU-A. При наличии наблюдений AMSU-A эффект от MTBЗА-ГЯ в обоих полушариях был нейтральным (не показано).

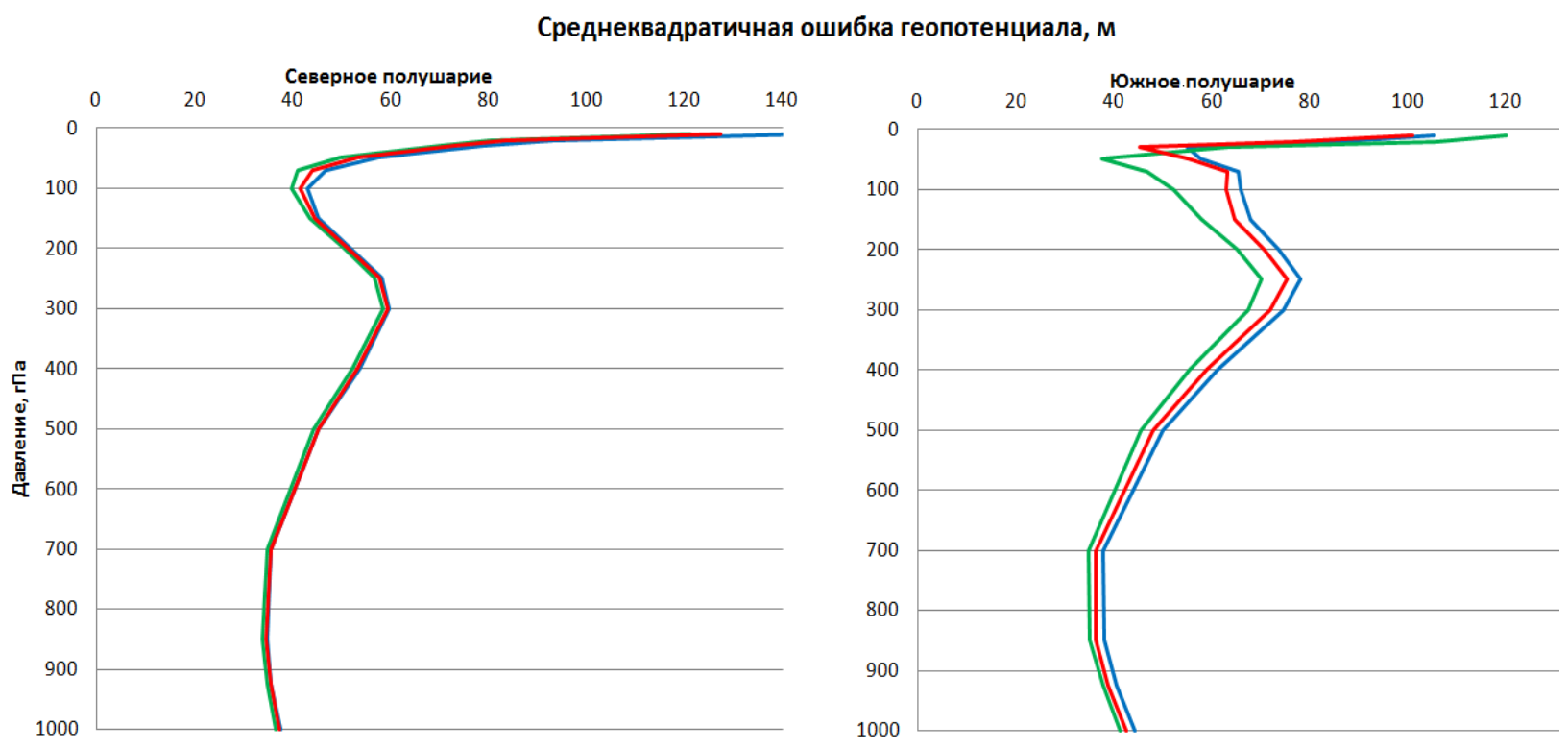

Рис. 1. Среднеквадратичная ошибка трехсуточного прогноза геопотенциала (м) по модели ПЛАВ [11]. Синяя линия - без использования микроволновых наблюдений, красная - с усвоением наблюдений MTBЗА-ГЯ, зеленая - с усвоением наблюдений AMSU-A спутника METOP-A

\section{Предварительный анализ точности данных измерений ИКФС-2}

Инфракрасный фурье-спектрометр ИКФС-2, установленный на борту спутника Метеор-М №2, имеет рабочий спектральный диапазон 5-15 мкм (2700 каналов), полосу обзора 1000 - 2500 км и пространственное разрешение в надире 35 км [12].

На рис.2 приведены оценённые нами (аналогично вышеизложенному для МТВЗА-ГЯ) смещения и стандартные отклонения для данных ИКФС-2 (слева) и IASI (справа). Эти результаты получены осреднением по 4-м суткам в сентябре 2018 г. Как данные ИКФС-2, так и данные IASI прошли коррекцию смещений типа аналогично данным МТВЗА-ГЯ и фильтрацию облачности согласно [13]. Кроме того, для получения более сопоставимых результатов данные IASI, имеющие более высокое пространственное разрешение (12 км), были осреднены по 4-м соседним т.н. IFOVs - это уменьшило их эффективное горизонтальное разрешение до 48 км, что соизмеримо (и даже ниже) разрешающей способности ИКФС-2. Отметим также, что учёт более высокого разрешения IASI в спектральной области может быть выполнен следуя [14]. 

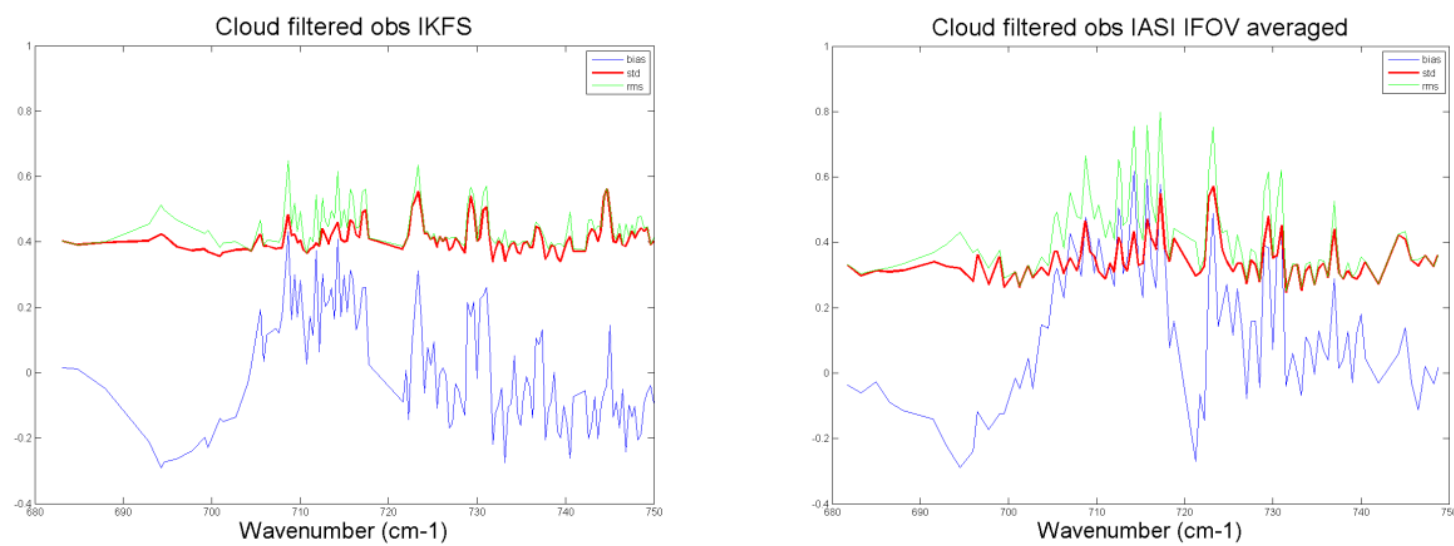

Рис. 2. Смещения (синие линии), стандартные отклонения (красные линии) и среднеквадратичные ошибки (зелёные линии) для данных ИКФС-2 (слева) и IASI (справа), ср. [12]

Видно, что точность данных ИКФС-2 практически не уступает таковой для IASI (считающегося «эталонным» инструментом).

\section{Первые результаты усвоения данных измерений ИКФС-2}

Были проведены численные эксперименты (аналогичные вышеприведённым для МТВЗА-ГЯ) по усвоению в системе усвоения данных Гидрометцентра России данных наблюдений ИКФС-2. Результаты усвоения данных в 20 частотных каналах, равномерно выбранных в 15-мкм полосе поглощения углекислого газа (и чувствительных, преимущественно, к температуре воздуха в тропосфере), показаны на рис.3. Видно ощутимое повышение точности прогнозов в результате усвоения данных ИКФС-2.

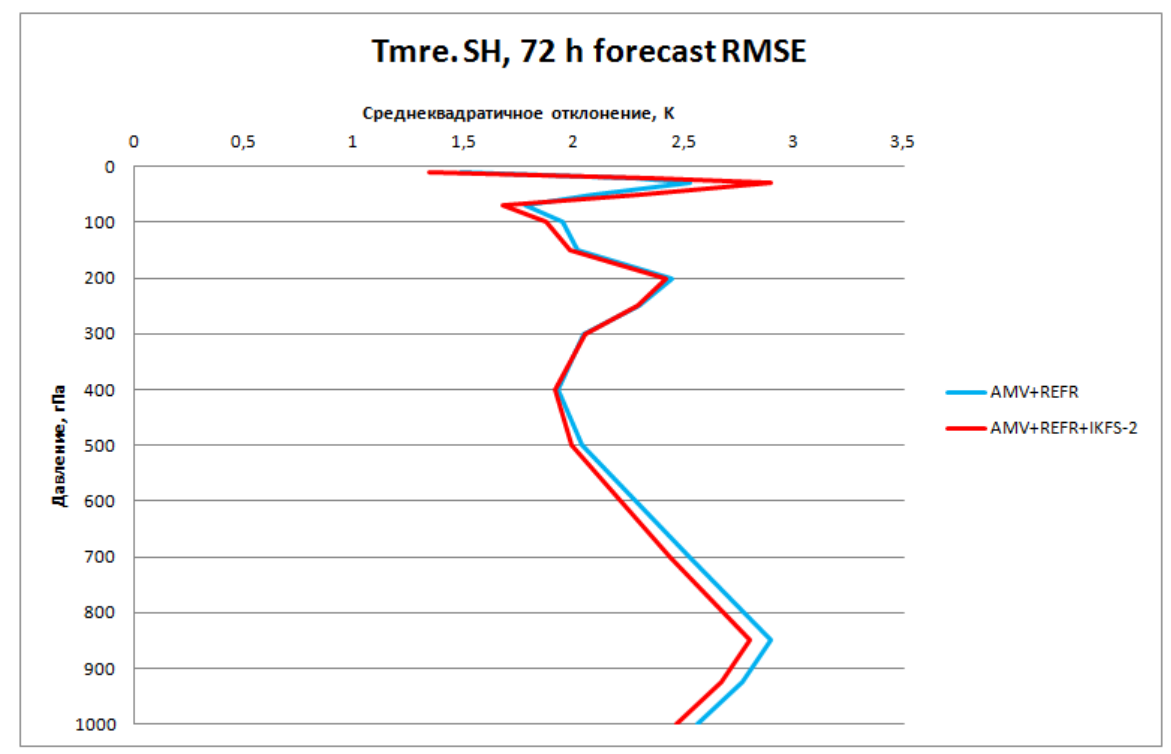

Рис. 3. Среднеквадратичная ошибка трехсуточного прогноза температуры (К). Синяя линия - без использования наблюдений ИКФС-2, красная линия - с использованием наблюдений ИКФС-2. Из остальных спутниковых наблюдений усваивались только спутниковые ветра (AMV) и профили коэффициента преломления воздуха (REFR) по данным радиозатменных наблюдений COSMIC и GRAS (см. [1] и ссылки там) 


\section{Заключение}

Дан краткий обзор современных методов оперативного усвоения данных для задачи глобального прогноза погоды, а также подходов к усвоению спутниковых радиационных наблюдений. Приведены результаты оценки точности данных измерений радиометра МТВЗА-ГЯ в температурных и влажностных каналах, а также измерений фурье-спектрометра ИКФС-2 в каналах полосы поглощения углекислого газа. Выявлен положительный эффект от использования данных наблюдений МТВЗА-ГЯ и ИКФС-2 в системе усвоения данных Гидрометцентра России.

\section{References}

[1] Gayfulin D., Tsyrulnikov M., Uspensky A., et al. The usage of MTVZA-GYa satellite microwave radiometer observations in the data assimilation system of the Hydrometcenter of Russia. Russian Meteorology and Hydrology, 2017, .42(9), pp.564-573, DOI: 10.3103/S1068373917090035.

[2] Tsyrulnikov M., Rakitko A. A hierarchical Bayes ensemble Kalman filter. Physica D: Nonlinear Phenomena, 2017, 338, pp.1-16, DOI: 10.1016/j.physd.2016.07.009.

[3] Gorin V.E. and Tsyrulnikov M.D. Estimation of multivariate observation-error statistics for AMSU-A data. Monthly Weather Review, 2011, 139(12), pp.3765-3780, DOI: 10.1175/2011MWR3554.1.

[4] Weston, P.P., Bell, W. and Eyre, J.R. Accounting for correlated error in the assimilation of high-resolution sounder data. Quarterly Journal of the Royal Meteorological Society, 2014, 140(685), pp.2420-2429, DOI: 10.1002/qj.2306.

[5] Gayfulin D., Tsyrulnikov M., Uspensky A. Assessment and Adaptive Correction of Observations in Atmospheric Sounding Channels of the Satellite Microwave Radiometer MTVZA-GY. Pure and Applied Geophysics, 2018, 175(10), pp. 3653-3670, DOI: 10.1007/s00024-018-1917-7.

[6] Saunders R., Matricardi M., Brunel P. An improved fast radiative transfer model for assimilation of satellite radiance observations. Quarterly Journal of the Royal Meteorological Society, 1999, 125(556), pp.1407-1425, DOI: 10.1002/qj.1999.49712555615.

[7] Geer A.J., Lonitz, K., Weston, P., et al. All-sky satellite data assimilation at operational weather forecasting centres. Quarterly Journal of the Royal Meteorological Society, 2018, 144(713), pp.1191-1217, DOI:

10.1002/qj.3202.

[8] Harris B.A., Kelly G. A satellite radiance-bias correction scheme for data assimilation. Quarterly Journal of the Royal Meteorological Society, 2002, 127(574), pp. 1453-1468, DOI: 10.1002/qj.49712757418.

[9] Dee D.P., Uppala, S. Variational bias correction of satellite radiance data in the ERA-Interim reanalysis. Quarterly Journal of the Royal Meteorological Society, 2009, 135(644), pp.1830-1841, DOI: 10.1002/qj.493.

[10] Asmus A.A., V. A. Zagrebaev, L. A. Makridenko et al. Meteorological satellites based on Meteor-M polar orbiting platform, Russian Meteorology and Hydrology, 2014, 39(12), pp.787-794.

[11] Tolstykh M.A., Geleyn J.F., Volodin E.M. et al. Development of the multiscale version of the SL-AV global atmosphere model. Russian Meteorology and Hydrology, 2015, 40(6), pp.374-382, DOI:

$10.3103 / \mathrm{S} 1068373915060035$.

[12] Golovin Yu. M., Kozlov D. A., Rublev A.N. et al. Infrared Fourier transform spectrometer IKFS-2: Results of operation onboard the meteorological satellite "METEOR-M" N2. Issledovanie Zemli iz Kosmosa 2017, 4, pp.88-100. DOI: 10.7868/S0205961417040091. (In Russian).

[13] McNally A. P., Watts P. D. A cloud detection algorithm for high-spectral-resolution infrared sounders. Quarterly Journal of the Royal Meteorological Society, 129(595), pp.3411-3423, DOI: 10.1256/qj.02.208. 
[14] Kozlov D.A., Timofeev Yu.M., Polyakov A.V. et al. Method of recalculation of thermal radiation spectra of different spectral resolutions for intercomparison of infrared onboard Fourier spectrometers. Sovremennye

Problemy Distantsionnogo Zondirovaniya Zemli iz Kosmosa, 2018, 15(1), pp. 52-60. DOI: 10.21046/2070-74012018-15-1-52-60. (In Russian). 